\title{
Obstruction of spreading droplets by non-deformable objects
}

\author{
Marcus Horwich*1, Yannis Hardalupas ${ }^{1}$ \\ ${ }^{1}$ Department of Mechanical Engineering, Imperial College London, U.K. \\ ${ }^{*}$ Corresponding author email: m.horwich17@imperial.ac.uk
}

\begin{abstract}
In this study, we investigate the impact of a droplet onto a solid surface in the vicinity of a sessile solid cylinder. Water droplets impacted an acrylic surface with Weber numbers in the range of $406-616$. The radius of the cylinder was varied $(0.52,0.28$ and $0.23 \mathrm{~mm}$, leading to ratios to the droplet radius of $0.12-0.39$ ) as well as the distance between the impact point and the cylinder $(1.75-10.57 \mathrm{~mm}$ leading to ratios to the droplet radius of $1.26-5.53)$. High speed cameras were used to capture the droplet temporal evolution and image analysis software used to extract features such as maximum spreading radius, numbers of corrugations and the behaviour of ligament length along the rim at the circumference of the lamella. We observe a dry ' $V$-shaped' area behind the cylinder and discuss the conditions under which the lamella is able to re-join around the rear of the cylinder.
\end{abstract}

\section{Keywords}

Droplet impact, Non-isolated, obstruction, sessile solid.

\section{Introduction}

Droplet impacts onto surfaces are ubiquitous in both industry and nature, from inkjet printing, fuel spray in internal combustion engines, agricultural herbicide spraying and spray coating to common raindrops falling and sneezing. Much effort has been made to understand and be able to control the outcomes of these impact events.

Upon impacting a solid surface, with a thin film, the fluid in a droplet is forced outwards into an expanding lamella. If the energy of the impact is large enough, a corona is formed, with a vertical sheet that expands radially with smaller secondary droplets ejected from the free rim. Yarin and Weiss [1] examined theoretically droplet impact in this scenario and first proposed a relationship for the rate at which the radial position of the crown rim evolved, finding the nondimensional spreading diameter was proportional to the root of dimensionless time $\left(r_{\max } / r \sim t^{1 / 2}\right)$, with the constant of proportionality, still debated. The process of droplet impacts on surfaces with liquid films is better understood than those on dry surfaces, with the number of possible outcomes of a droplet impact dry-walls larger than that of a wetted surface. This is due to extra surface properties that are prevalent in the dry wall case, which are not as important in the wetted wall condition, such as wettability (hydrophobic/hydrophilic) and surface roughness [2]. The impact of a droplet on a dry-wall can lead to into six possible scenarios (a) Prompt splash; (b) Corona splash; (c) Receding breakup; (d) rebound; (e) Partial rebound; (f) deposition [3]. The boundaries between the point of splashing and deposition have been investigated by Stow and Hadfield [4] as well as Mundo et al. [5]. Mainly flat walled surfaces have been studied, however, several authors such as Charalampous and Hardalupas [6] and Bakshi et al. [7] have investigated impacts onto curved surfaces.

Common non-dimensional parameters pertinent to droplet impact studies, are the Weber number, We (Equ. (1)) and the Ohnesorge number, Oh (Equ. (2)).

$$
W e=\frac{\rho v^{2} 2 r}{\sigma}
$$




$$
O h=\frac{\mu}{\sqrt{\rho \sigma 2 r}}
$$

Many authors have looked at the effect of these on the maximum spreading diameter such as Scheller and Bousfield [8] who found an empirical relationship between the non-dimensional maximum spreading radius, $W e$ and $O h$ and is given by: $r_{\max } / r=0.61(W e / O h)^{1 / 6}$. Other interesting phenomena occur during the spreading phase of a droplet impact such as fingering, where fingers/corrugations appear along the edge of the expanding rim. Marmanis and Thoroddsen [9] examined the number of corrugations on the edge of the expanding lamella and were able to determine that the number of corrugations was given by $N=C(\mathrm{We} / \mathrm{Oh})^{3 / 8}$ with Range and Feuillebois [10] determining $C$ to be dependent on surface roughness and wettability.

The majority of the research into droplet impacts has taken place with droplets impacting a surface in isolation. However, single, isolated, droplet impacts onto regular flat surfaces do not occur frequently in the real world, where there may be complex geometries with protrusions or other deformable obstacles which can interact with the edge of the lamella. Wang and Bourouiba [11] looked into the outcomes of droplet impacts in the presence of another sessile liquid droplet and discovered a new mode of secondary droplet formation, namely crescent-moon fragmentation, where secondary droplets are ejected at $W e$ numbers that would not produce secondary droplets during isolated impacts.

This work presents a study into the impact of droplets in the vicinity of a solid cylindrical object, and quantifies the effects on the subsequent lamella formation.

\section{Experimental Arrangement}

Experiments were conducted with water (fluid properties shown in Table 1) to examine the characteristics of a droplet impact in the vicinity of a solid obstruction. The impact substrate was chosen to be a smooth acrylic surface, onto which sessile solid cylinders could be attached. Three solid cylinders were used in the experiment with radii $r_{c y l}$ of $0.53,0.28$ and $0.23 \mathrm{~mm}$ leading to non-dimensional ratios $r_{c y l} / \mathrm{r}$ of $0.12-0.39$, where $r$ is the droplet radius. The experimental set-up is shown in Figure 1.

A blunt tip needle was clamped $0.75 \mathrm{~m}$ above the surface and a droplet with radius $r$ was formed at its end using a syringe pump attached to a fine stepper motor controlled by an Arduino Uno board and motor shield. The droplet was allowed to fall under its own gravity until impacting the acrylic surface below in the vicinity of the cylindrical sessile solid. The size of the falling droplet, the impact distance, $d$, between the falling droplet and the cylinder and the cylinder radius, $r_{c y l}$ were varied during the experiment and summarised in Table 2.

Upon impact with the surface, the droplet expands into a thin liquid sheet with maximum radius $r_{\text {max }}$. Two high-speed cameras with macro-lenses (f-150 and f-200) were used in order to capture simultaneously both the side-view and bottom-view of the droplet impact and record the temporal evolution of the droplet diameter and velocity and various spreading characteristics that are shown in Figure 2. The resolution of the recorded images was 29 $\mu \mathrm{m} /$ pixel and $62 \mu \mathrm{m} /$ pixel for side and bottom-views respectively. These features were then extracted from the high-speed video images using Python and the OpenCV image analysis processing toolkit.

The parameters that were investigated during the experiment are shown in Figure 2. The radius of the cylinder obstruction, placed vertically on the flat surface, is shown as $r_{c y l}$. After a droplet of radius $r$ impacts the surface, the lamella spreads and expands to a maximum 
radius, shown as $r_{\text {max }}$. The distance $d$ can be seen as the distance between the point of droplet impact and the centre axis of the vertical cylinder. It should be noted that if $r_{\max }$ is greater than $d$, no interaction occurs. $N$ is the number of corrugations around the lamella (measured by doubling the number of corrugations over half of the lamella unaffected by the cylinder's presence), with the wavelength $\lambda$ equal to the average wavelength between these corrugations.

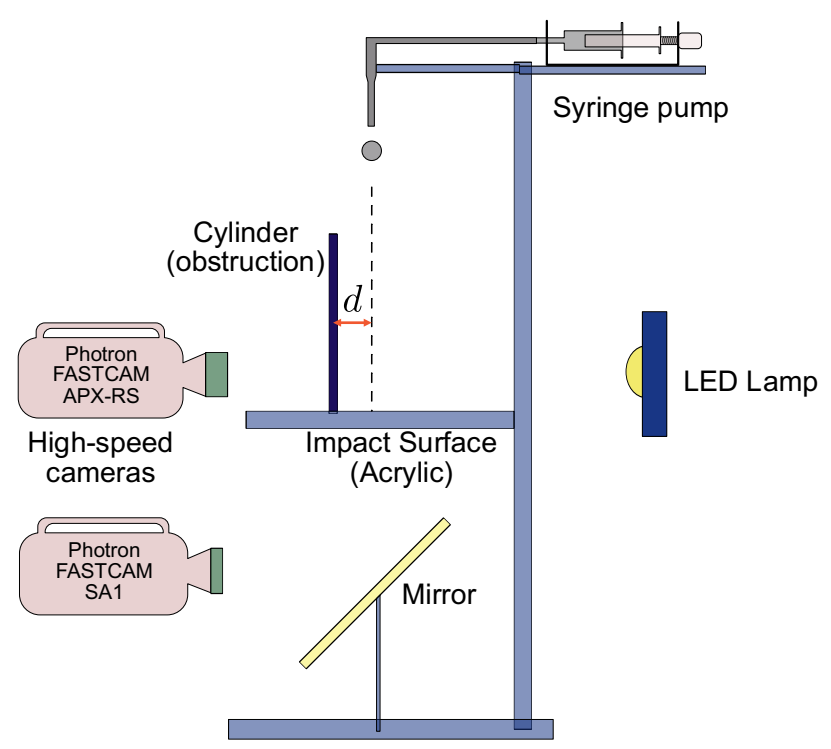

Figure 1. Schematic of the Experiment set-up

Table 1 - Droplet fluid properties

\begin{tabular}{c|cccc}
\hline Fluid & $\begin{array}{c}\text { Density } \\
\rho\left[\times 10^{3} \mathrm{~kg} \mathrm{~m}^{-3}\right]\end{array}$ & $\begin{array}{c}\text { Viscosity } \\
\mu\left[\times 10^{-3} \mathrm{Pas}\right]\end{array}$ & $\begin{array}{c}\text { Surface Tension } \\
\sigma\left[\times 10^{-3} \mathrm{~N} \mathrm{~m}^{-1}\right]\end{array}$ & $\begin{array}{c}\text { Equilibrium } \\
\text { contact angle } \theta_{e}\left[{ }^{\circ}\right]\end{array}$ \\
\hline Water & 1.000 & 1.0 & 72 & 68.2 (acrylic)
\end{tabular}

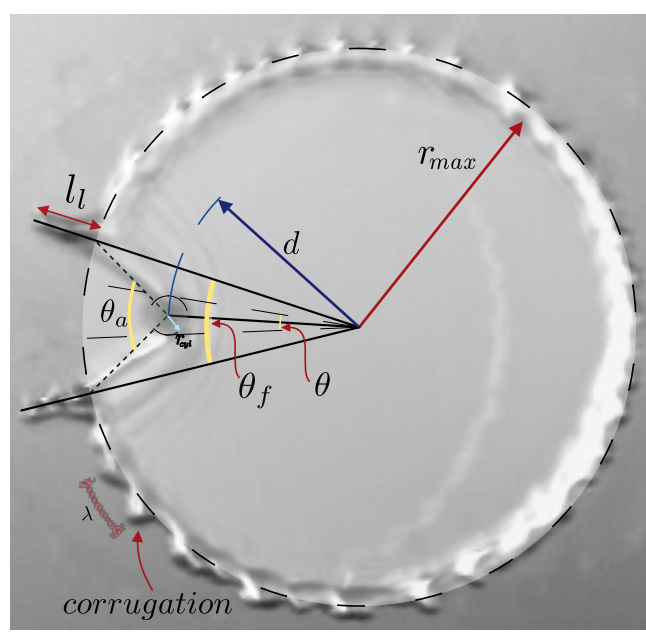

(a)

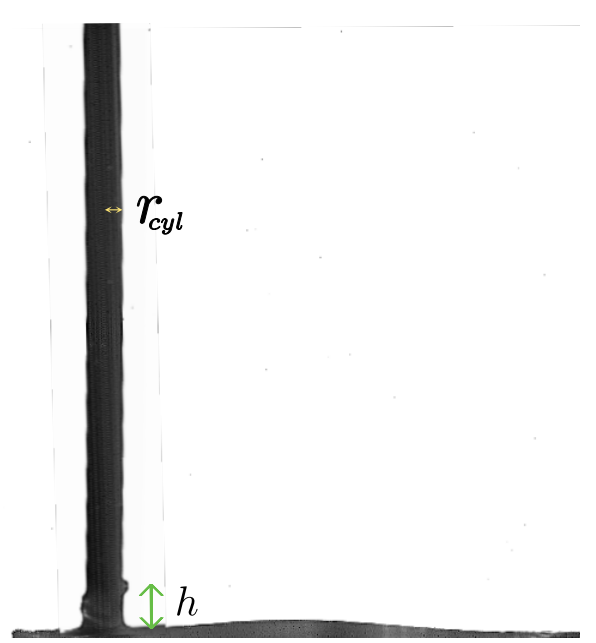

(b)

Figure 2. Schematic of relevant spreading parameters during droplet impact on a surface, which interacts with a cylindrical obstacle. (a), bottom-view, (b), side-view 
It can be seen from Figure 2(a) that a ' $V$-shaped' sector is removed from the lamella, and there is an area of dry surface behind the cylinder, bounded by two extended ligaments of length $l_{l}$. Three angles were measured; $\theta$ which is the angle created from two lines tangential to the cylinder passing through the impact point; $\theta_{f}$ which is the angle of the sector of the lamella between the two extended ligaments; and $\theta_{a}$, the angle between the two extended ligaments measured from the centre of the cylinder. Figure 2(b) shows the splashing height $h$ of the lamella against the side of the cylinder; however, quantitative results about $h$ are not presented here.

Table 2 - Experimental Conditions considered during the current study

\begin{tabular}{|c|c|c|c|c|c|c|c|c|}
\hline Case & $r[\mathrm{~mm}]$ & $W e$ & $r_{c y l}[\mathrm{~mm}]$ & $r_{c y l} / r$ & $d[\mathrm{~mm}]$ & $d / r$ & $r_{\max }[\mathrm{mm}]$ & $N$ \\
\hline 1 & \multirow{3}{*}{$\begin{array}{c}1.91 \pm \\
0.02\end{array}$} & \multirow{3}{*}{$\begin{array}{c}616 \pm \\
20\end{array}$} & 0.53 & 0.28 & \multirow{3}{*}{$\begin{array}{l}3.01- \\
10.57\end{array}$} & \multirow{3}{*}{$\begin{array}{c}1.56- \\
5.53\end{array}$} & \multirow{3}{*}{$\begin{array}{c}9.89 \pm \\
0.13\end{array}$} & \multirow{3}{*}{$\begin{array}{c}45 \pm \\
2\end{array}$} \\
\hline 2 & & & 0.28 & 0.15 & & & & \\
\hline 3 & & & 0.23 & 0.12 & & & & \\
\hline 4 & \multirow{3}{*}{$\begin{array}{c}1.53 \pm \\
0.01\end{array}$} & \multirow{3}{*}{$\begin{array}{c}520 \pm \\
9\end{array}$} & 0.53 & 0.35 & \multirow{3}{*}{$\begin{array}{c}1.93- \\
7.93\end{array}$} & \multirow{3}{*}{$\begin{array}{c}1.26- \\
5.18\end{array}$} & \multirow{3}{*}{$\begin{array}{c}7.53 \pm \\
0.15\end{array}$} & \multirow{3}{*}{$\begin{array}{c}42 \pm \\
3\end{array}$} \\
\hline 5 & & & 0.28 & 0.18 & & & & \\
\hline 6 & & & 0.23 & 0.15 & & & & \\
\hline 7 & \multirow{3}{*}{$\begin{array}{c}1.35 \pm \\
0.01\end{array}$} & \multirow{3}{*}{$\begin{array}{c}406 \pm \\
6\end{array}$} & 0.53 & 0.39 & \multirow{3}{*}{$\begin{array}{c}1.75- \\
7.35\end{array}$} & \multirow{3}{*}{$\begin{array}{c}1.30- \\
5.44\end{array}$} & \multirow{3}{*}{$\begin{array}{c}6.50 \pm \\
0.06\end{array}$} & \multirow{3}{*}{$\begin{array}{c}39 \pm \\
4\end{array}$} \\
\hline 8 & & & 0.28 & 0.21 & & & & \\
\hline 9 & & & 0.23 & 0.17 & & & & \\
\hline
\end{tabular}

48 sets of videos were considered for each case, with the standard deviation of the measured quantities shown in Table 2. This demonstrates that the fluctuations of the measured quantities remain small.

\section{Results and Discussion}

Two examples of droplet impacts are shown in Figure 3. The top set of images show the side and bottom views for an impact for condition 2 from Table 2, with $d / r_{\max }=0.72$ and $r_{c y l}=0.23 \mathrm{~mm}$. Due to interactions between the droplet and the air, the impacting droplet was not always completely spherical at the time of impact. From the time evolution, it can be seen that once the lamella expands on the surface, corrugations begin to appear as expected, and then the edge of the lamella interacts with the cylinder. After the initial droplet impact, the lamella continues expanding to its maximum radius $r_{\text {max }}$. However, a dry ' $V$ shaped' segment (with the base located at the cylinder) is removed from the lamella in the region behind the cylinder, with two long ligaments formed at the tips of the ' $\mathrm{V}$. The lower set of images show an impact for Condition 1, with $d / r_{\max }=0.38, r_{c y l}=0.28 \mathrm{~mm}$. The droplet impact begins the same as in the previous example. However, in this case, after the initial impact, the lamella is able to re-join around the rear of the cylinder. It is unable to recover all the missing area lost in the previous case and leads to a ' $U$-shaped' dry-region behind the cylinder, again with the tips of the ' $U$ ' surrounded by two extended ligaments. For the analysis, the spreading diameter was measured using a Random Sample Consensus (RANSAC) algorithm on the bottom-view images in order to determine the spreading radius, $r_{\text {max }}$ for the three $W e$ numbers of the experiment, as shown in Table 2. By binarizing the image and using edge detecting kernels, random samples of points were repeatedly selected in order to find a circle to which the data best fit. Scheller and Bousfield [8] determined a relationship between spreading diameter and $W e$ in the form $r_{\text {max }} / r=0.61 O h^{-1 / 6} W e^{1 / 6}$. By fitting the 
data with this scaling of $W e$ and $O h$, our data was better fit by $r_{\max } / r=0.63 O h^{-1 / 6} W e^{1 / 6}$. For this investigation, $O h^{-1 / 6}$ has an almost constant value of 2.8 and the relationship can therefore be expressed as $r_{\max } / r=1.76 \mathrm{~W} e^{1 / 6}$.

The dependency of the number of corrugations on $W e$ was examined and is also shown in Table 2. These were in good agreement with the scaling found by Marmanis and Thoroddsen [9], where $N \sim W e^{3 / 8}$, with the constant of proportionality in this case being 4.1, i.e., $N=4.1 \mathrm{~W} e^{3 / 8}$. From this, the wavelength $\lambda$ (Figure 2) can be determined by dividing the perimeter length by the number of corrugations, $\left(2 \pi r_{\max } / N\right)$ giving $1.38 \mathrm{~mm}, 1.13 \mathrm{~mm}$, $1.05 \mathrm{~mm}$ for the three $W e$ numbers of $616,520,406$ respectively. The radii of the cylinders are therefore shorter than the wavelength of the corrugations.

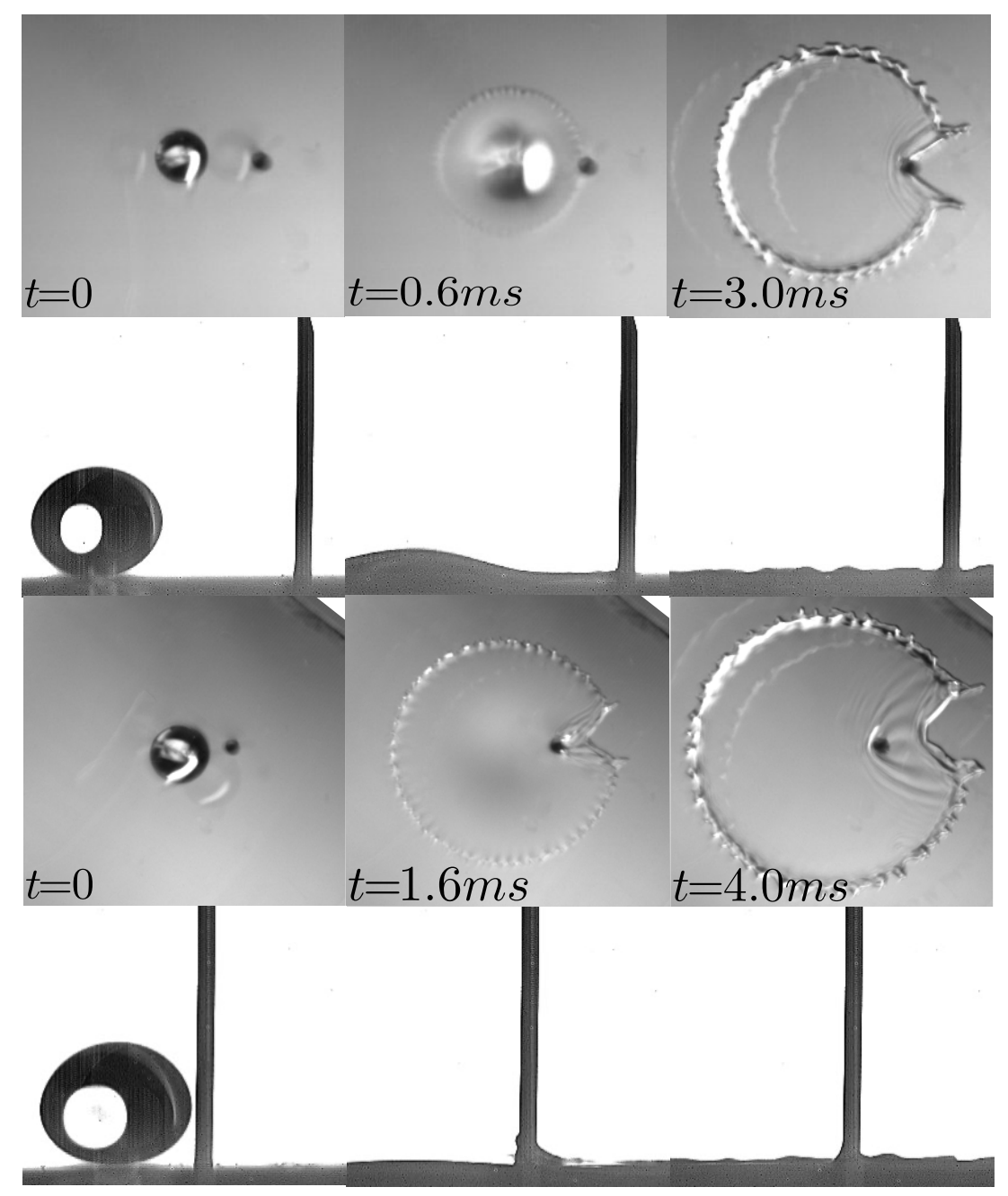

Figure 3. Example droplet impacts on flat surfaces with cylindrical obstacles. Side and bottom views at different times after the start of the impact for different droplet sizes and distances to the obstacle. Top images: Case 6, $d / r_{\max }=0.72, r_{c y l}=0.23 \mathrm{~mm}$. Bottom: Case 2, $d / r_{\max }=0.38, r_{c y l}=0.28 \mathrm{~mm}$

The opening angles $\theta_{f}$ and $\theta_{a}$ (Figure 2) were measured and plotted against $d / r_{\text {max }}$ in Figure 4. $\theta_{f}$ follows a negative linear relationship with $d / r_{\text {max }}$. By geometry, it can be seen that the two opening angles are related by the equation: $\tan \left(\theta_{a} / 2\right)=\sin \left(\theta_{f} / 2\right) /\left[\cos \left(\theta_{f} / 2\right)-d / r_{\max }\right]$, hence the non-linear increase of $\theta_{a}$ with $d / r_{\max }$. As $d / r_{\max } \rightarrow 0 ; \theta_{a} \rightarrow \theta_{f}$. As shown above, $r_{\text {max }} \sim W e^{1 / 4}$, therefore, for low values of $d / r_{\max }$, the impact can be thought of as having a 
large ratio of inertial to surface tension forces and a small distance to the cylinder. As the inertial forces dominate the surface tension forces, the rim of the lamella is able to deflect more sharply around the cylinder, decreasing $\theta_{a}$.
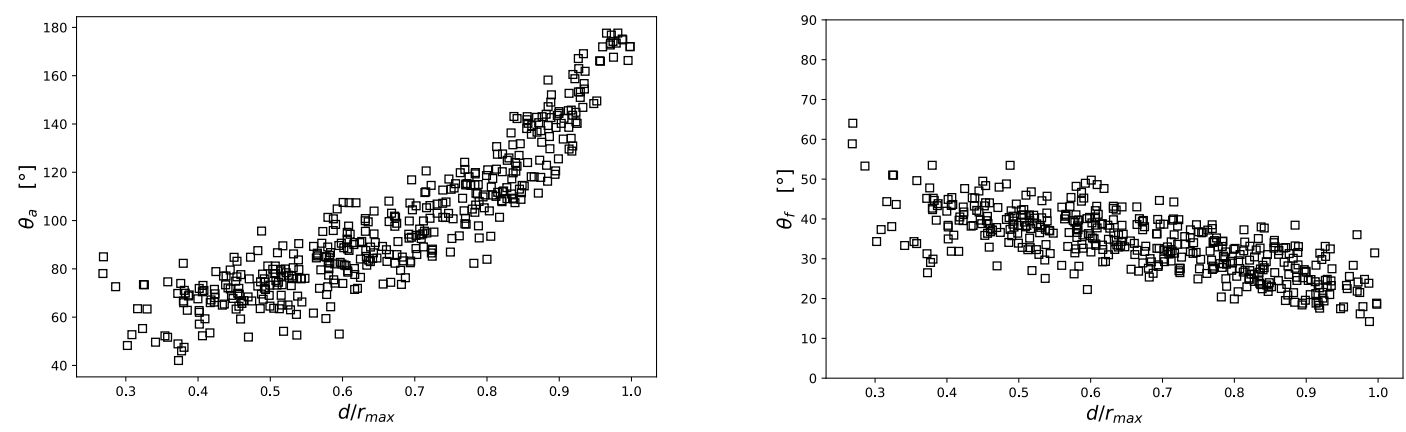

Figure 4. (a) Opening angle $\theta_{a}$, (b) Opening angle $\theta_{f}$ as a function of non-dimensional distance. Definitions of the relevant angles are shown in Figure 2.

By observing the schematic of the droplet impact in Figure 2, it was posited that the missing lamella fluid from the ' $V$-shaped' area behind the cylinder, is forced into the two extended ligaments. By equating the volume of these ligaments with the ' $V$-shaped' region, a prediction for the ligament length could be established. From geometric inspection, the area of the ' $V$ shaped' region $A_{m}$ is approximated by Equ. (3), which can then be multiplied by the thickness of the lamella, $\delta$, to give the volume of the ' $V$-shaped' region. $\delta$ is calculated by equating the volume of the impacting droplet with the volume of the lamella [11].

$$
\begin{aligned}
& A_{m} \approx\left(r_{\max }-d\right) r_{\max } \sin \left(\frac{\theta_{f}-\theta}{2}\right)+\frac{\theta}{2}\left(r_{\max }^{2}-d^{2}\right) \\
& \delta=\frac{4 r^{3}}{3 r_{\max }^{2}}
\end{aligned}
$$

The ligaments were approximated by semi-cylindrical segments with chord length $w_{c}=\lambda / 2=$ $\left(\pi r_{\max } / N\right)$, and forming an angle with the surface of $\theta_{e}$ (equilibrium contact angle). The volume of a single ligament $V_{l}$, can be written in the form $V_{l}=C_{s} w_{c}{ }^{2} l_{l}$ as shown in Equ. (5), where $C_{s} w_{c}{ }^{2}$ is the cross-sectional area of the semi-cylindrical segment. For water on acrylic (Table 1), $C_{s}=0.25$. Equating the volumes of the two ligaments and the ' $V$-shaped' region, Equ. (6) is obtained and finally, substituting the previous relationships for $C_{s}$ as well as $N$ and $r_{\text {max }}$ in terms of $W e$, we arrive at Equ. (7).

$$
\begin{aligned}
& V_{l}=\frac{1}{4}\left(\theta_{e} \csc ^{2}\left(\theta_{e}\right)-\cot \left(\theta_{e}\right)\right) w_{c}^{2} l_{l} \\
& \frac{l_{l}}{r_{\max }}=\frac{2}{3 C_{s}}\left(\frac{N}{\pi}\right)^{2}\left(\frac{r}{r_{\max }}\right)^{3}\left[\left(1-\frac{d}{r_{\max }}\right) \sin \left(\frac{\theta_{f}-\theta}{2}\right)+\frac{\theta}{2}\left(1+\frac{d}{r_{\max }}\right)\left(1-\frac{d}{r_{\max }}\right)\right]
\end{aligned}
$$




$$
\frac{l_{l}}{r_{\max }}=\frac{2}{3 \pi^{2}(0.25)} \frac{(4.1)^{2}}{(1.76)^{3}} W e^{1 / 4}\left[\left(1-\frac{d}{r_{\max }}\right) \sin \left(\frac{\theta_{f}-\theta}{2}\right)+\frac{\theta}{2}\left(1+\frac{d}{r_{\max }}\right)\left(1-\frac{d}{r_{\max }}\right)\right]
$$

The experimental results are plotted in Figure 5 with Equ. (7) plotted as a straight line with gradient equal to $0.85\left(\frac{2}{3 \pi^{2}(0.25)} \frac{(4.1)^{2}}{(1.76)^{3}}\right)$ to evaluate the validity of the model. The results were grouped by the ratio of impact distance to spreading radius $d / r_{\max }$. The relationship appears to describe well the behaviour for large values of $d / r_{\max }$, but breaks down as the ratio decreases. One explanation for this can be seen in Figure 3. At large values of $d / r_{\text {max }}$, a well defined ' $V$-shaped' area is removed from the lamella, while, as $d / r_{\text {max }}$ decreases, the lamella re-joins around the rear of the cylinder, and hence, there is fluid in the ' $V$-shaped' area and therefore, the geometric approximation overestimates the length of the ligaments. It is noted that Equ. (7) fails to scale the behaviour for low $d / r_{\text {max }}$ values because it does not consider the re-joining of the lamella in the wake of the cylinder. The theory is currently re-considered to establish a new scaling approach that can address this additional behaviour.

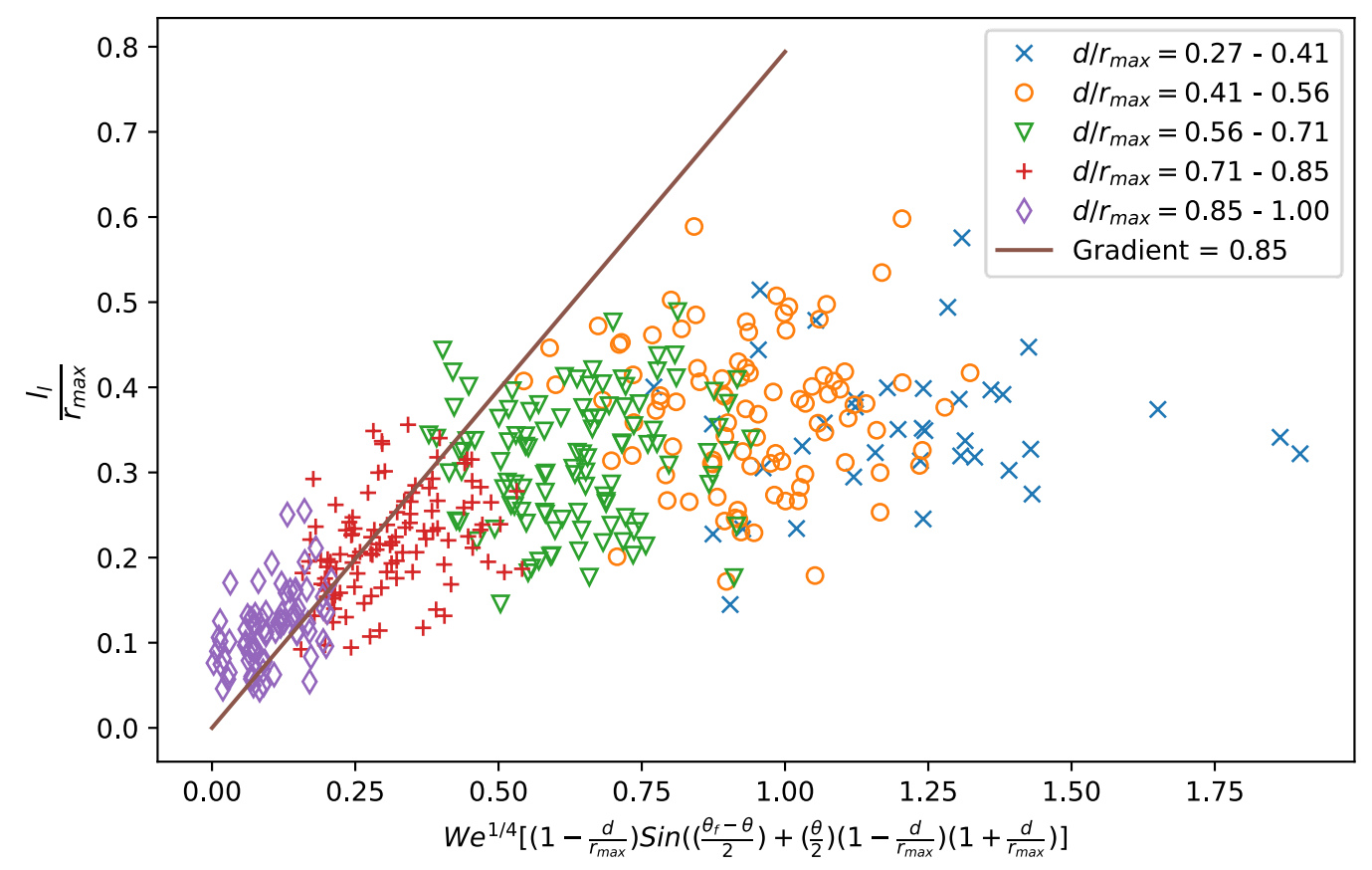

Figure 5. Ligament length for different distances between droplet impact and obstacle locations is plotted according to the derived Equation (7)

\section{Conclusions}

A study was carried out into the impact of a droplet on a solid surface in the vicinity of a sessile solid cylinder with radius smaller than the wavelength of the corrugation pattern around the lamella. It was found that the properties of the spreading lamella, such as number of corrugations and maximum spreading diameter, were in good agreement with previous research. We observed the ' $V$-shaped' dry region of the lamella behind the cylinder as seen in [11] for large values of $d / r_{\max }$ and examined the behaviour of the ligament length. We identified a new phenomenon of the re-joining of the lamella behind the cylinder as $d / r_{\max }$ is reduced, which affects the ability of existing theoretical descriptions to describe the experimental observations. 


\section{Acknowledgments}

This project was supported by the UK Engineering and Physical Sciences Research Council (EPSRC) through the Centre for Doctoral Training in fluid dynamics across scales (grant EP/L016230/1).

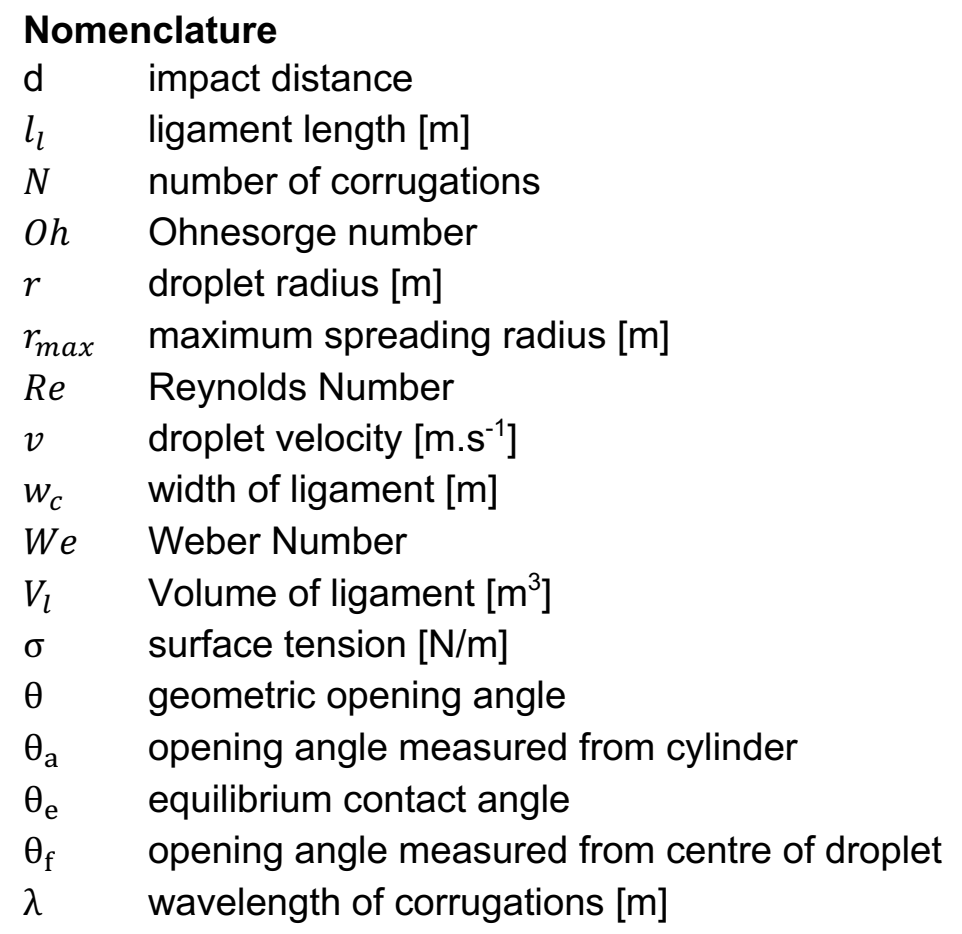

\section{References}

[1] Yarin, A. L., and Weiss, D. A., 1995, "Impact of Drops on Solid Surfaces: Self-Similar Capillary Waves, and Splashing as a New Type of Kinematic Discontinuity," J. Fluid Mechanics, 283(1), p. 141.

[2] Yarin, A. L., 2006, "DROP IMPACT DYNAMICS: Splashing, Spreading, Receding, Bouncing...," Annual Review of Fluid Mechanics, 38(1), pp. 159-192.

[3] Rioboo, R., Tropea, C., and Marengo, M., 2001, "OUTCOMES FROM A DROP IMPACT ON SOLID SURFACES," Atomization and Sprays, 11(2), p. 12.

[4] Stow, C. D., and Hadfield, M. G., 1981, "An Experimental Investigation of Fluid Flow Resulting from the Impact of a Water Drop with an Unyielding Dry Surface," Proceedings of the Royal Society A: Mathematical, Physical and Engineering Sciences, 373(1755), pp. 419-441.

[5] Mundo, C., Sommerfeld, M., and Tropea, C., 1995, "Droplet-Wall Collisions: Experimental Studies of the Deformation and Breakup Process," International Journal of Multiphase Flow, 21(2), pp. 151-173.

[6] Charalampous, G., and Hardalupas, Y., 2017, "Collisions of Droplets on Spherical Particles," Physics of Fluids, 29(10), p. 103305.

[7] Bakshi, S., Roisman, I. V., and Tropea, C., 2007, "Investigations on the Impact of a Drop onto a Small Spherical Target," Physics of Fluids, 19(3), p. 032102.

[8] Scheller, B. L., and Bousfield, D. W., 1995, "Newtonian Drop Impact with a Solid Surface," AIChE Journal, 41(6), pp. 1357-1367.

[9] Marmanis, H., and Thoroddsen, S. T., 1996, "Scaling of the Fingering Pattern of an Impacting Drop," Physics of Fluids, 8(6), pp. 1344-1346.

[10] Range, K., and Feuillebois, F., 1998, "Influence of Surface Roughness on Liquid Drop Impact," Journal of Colloid and Interface Science, 203(1), pp. 16-30.

[11] Wang, Y., and Bourouiba, L., 2018, "Non-Isolated Drop Impact on Surfaces," J. Fluid Mech., 835, pp. 24-44. 